Mathematical Modelling and Analysis

Volume 20 Number 1, January 2015, 30-52

http://dx.doi.org/10.3846/13926292.2015.1000411

(c) Vilnius Gediminas Technical University, 2015
Publisher: Taylor\&Francis and VGTU

http://www.tandfonline.com/TMMA

ISSN: $1392-6292$

eISSN: $1648-3510$

\title{
Periodic and Chaotic Orbits of a Neuron Model
}

\author{
Aija Anisimova ${ }^{a}$, Maruta Avotina $^{a}$ and Inese Bula ${ }^{a, b}$ \\ ${ }^{a}$ University of Latvia \\ Zellu 8, LV-1002 Rīga, Latvia \\ ${ }^{b}$ Institute of Mathematics and Computer Science of University of Latvia \\ Raiņa bulv. 29, LV-1048 Rīga, Latvia \\ E-mail: aija-anisimova@inbox.1v \\ E-mail: marutaavotina@inbox.1v \\ E-mail(corresp.): ibula@lanet.lv
}

Received September 30, 2013; revised December 1, 2014; published online January 15, 2015

\begin{abstract}
In this paper we study a class of difference equations which describes a discrete version of a single neuron model. We consider a generalization of the original McCulloch-Pitts model that has two thresholds. Periodic orbits are investigated accordingly to the different range of parameters. For some parameters sufficient conditions for periodic orbits of arbitrary periods have been obtained. We conclude that there exist values of parameters such that the function in the model has chaotic orbits. Models with chaotic orbits are not predictable in long-term.
\end{abstract}

Keywords: chaotic mapping, dynamical system, iterative process, nonlinear problem, stability.

AMS Subject Classification: 39A10; 39A23; 92B20.

\section{Introduction}

The number of neurons in the human brain is 86 billion [4]. Although the proverbial forest is not seen because of the trees, a detailed study of single neurons is both a significant and interesting subject itself, and it is not necessary to understand the macroscopic dynamics and function of neural networks. In [19] a delay differential equation

$$
x^{\prime}(t)=-g(x(t-\tau))
$$

is used as a model for a single neuron with no internal decay where $g: R \rightarrow R$ is a signal (or activation, or amplification) function and $\tau \leq 0$ is a synaptic transmission delay. Equation (1.1) as a differential equation has been analysed in many papers, for example, $[6,7,13,17]$ and has applications in biology, economics, ecology, engineering. From (1.1) we obtain a model for a single neuron with no internal decay as the following equation

$$
x^{\prime}(t)=-g(x([t]))
$$


where $[t]$ denotes a greatest integer function. When we integrate (1.2) from $n$ to $t \in[n, n+1[$ we get

$$
x(t)=x(n)-\int_{n}^{t} g(x([s])) d s=x(n)-g(x(n))(t-n) .
$$

Letting $t \rightarrow n+1$ and denoting $x(n)=x_{n}$, we obtain a difference equation

$$
x_{n+1}=x_{n}-g\left(x_{n}\right)
$$

The same as in $[20,22]$ we consider a single neuron model

$$
x_{n+1}=\beta x_{n}-g\left(x_{n}\right) \text {, }
$$

where $\beta$ is interpreted as an internal decay rate and $g$ is a signal function. Typical signal functions (activation functions, amplification functions or inputoutput functions) are step functions, piecewise linear functions or sigmoid functions.

Models involving a step signal function are referred as McCulloch-Pitts models, in recognition of the pioneering work of McCulloch and Pitts from 1943 (the function describes an all-or-none property of a neuron in the model of McCulloch-Pitts). A sigmoid function is the most common form of a signal function. It is defined as a strictly increasing smooth bounded function satisfying certain concavity and asymptotic properties.

In [22], Zhou considered a single neuron model (1.3) with a signal function in a very simple form

$$
g(x)= \begin{cases}1, & x \geq 0 \\ -1, & x<0\end{cases}
$$

In [3], the authors investigated a neuron model (1.3) with parameter $0<$ $\beta \leq 1$ and a signal function (a function that is a little bit similar to the sigmoid function in comparison with (1.4))

$$
g(x)=\left\{\begin{array}{ll}
-b, & x \leq-\alpha \\
-a, & -\alpha<x<0, \\
0, & x=0, \\
a, & 0<x<\alpha, \\
b, & \alpha \leq x
\end{array} \quad(b>a>0 \text { and } \alpha>0)\right.
$$

where $-\alpha, 0, \alpha$ are three thresholds. In [3] some results about the periodicity of solutions of difference equation (1.3) were proved. In the case when $\beta>1$ there are difficulties to study the behavior of solutions because there is a great number of periodic orbits. Therefore in this paper we consider a single neuron model (1.3) but we propose to look at a different step signal function with two thresholds:

$$
g(x)= \begin{cases}-a, & x \leq-\alpha \\ 0, & -\alpha<x<\alpha, \quad(a>0 \text { and } \alpha>0) \\ a, & \alpha \leq x\end{cases}
$$


Some results about the periodicity of solutions of difference equation (1.3) accordingly to $\beta$ and parameters $\alpha$ and $a$ of the signal function will be proved. Oscillations are temporal periodic changes in the state of a system. In nonlinear systems like brain, oscillations define a stable state. If $\beta>1$ then it is possible to find cases when the model has chaotic orbits.

\section{Basic Concepts and Definitions of Difference Equations}

To analyse the behavior of model (1.3) some basic concepts of difference equation theory (see $[9,10,12,22])$ are required.

We consider a first-order difference equation

$$
x_{n+1}=f\left(x_{n}\right), \quad n=0,1, \ldots,
$$

where $f: \mathbf{R} \rightarrow \mathbf{R}$ is a given function. A solution of equation (2.1) is a sequence $\left(x_{n}\right)_{n \in \mathbf{N}}$ that satisfies equation $(2.1)$ for all $n=0,1, \ldots$ If an initial condition $x_{0} \in \mathbf{R}$ is given, then the orbit $O\left(x_{0}\right)$ of a point $x_{0}$ is defined as a set of points

$$
O\left(x_{0}\right)=\left\{x_{0}, x_{1}=f\left(x_{0}\right), x_{2}=f\left(x_{1}\right)=f^{2}\left(x_{0}\right), x_{3}=f\left(x_{2}\right)=f^{3}\left(x_{0}\right), \ldots\right\} .
$$

Definition 1. A point $x_{s}$ is said to be a fixed point of the map $f$ or an equilibrium or a stationary state of equation (2.1) if $f\left(x_{s}\right)=x_{s}$.

Note that for a stationary state $x_{s}$ the orbit consists only of the point $x_{s}$.

DEFINITION 2. A stationary state of (2.1) is stable if

$$
\forall \varepsilon>0 \exists \delta>0 \forall x_{0} \in \mathbf{R} \forall n \in \mathbf{N} \quad\left|x_{0}-x_{s}\right|<\delta \Rightarrow\left|f^{n}\left(x_{0}\right)-x_{s}\right|<\varepsilon .
$$

Otherwise, the stationary state $x_{s}$ is called unstable.

DEFINITION 3. A stationary state $x_{s}$ of (2.1) is asymptotically stable if it is stable and attracting, i.e., it is stable and if there exists $\nu>0$ such that $\left|x_{0}-x_{s}\right|<\nu$ implies $\lim _{n \rightarrow \infty} f^{n}\left(x_{0}\right)=x_{s}$.

Definition 4. An orbit $O\left(x_{0}\right), x_{0} \in \mathbf{R}$, is said to be eventually stationary state to $x_{s}$ if

$$
\exists N \forall n \geq N \quad x_{n+1}=x_{n}=x_{s} .
$$

Definition 5. An orbit $O\left(x_{0}\right), x_{0} \in \mathbf{R}$, is said to be asymptotically stationary state to $x_{s}$ if $\lim _{n \rightarrow \infty} f^{n}\left(x_{0}\right)=x_{s}$.

Definition 6. An orbit $O\left(x_{0}\right)$ of the initial point $x_{0}$ of equation (2.1) is said to be periodic of period $p \geq 2$ if

$$
x_{p}=x_{0} \quad \text { and } \quad x_{i} \neq x_{0}, \quad 1 \leq i \leq p-1 .
$$

Definition 7. A periodic orbit $\left\{x_{0}, x_{1}, x_{2}, \ldots, x_{p-1}, \ldots\right\}$ of period $p$ is stable if each point $x_{i}, i=0,1, \ldots, p-1$, is a stable stationary state of the difference equation $x_{n+1}=f^{p}\left(x_{n}\right)$. A periodic orbit of period $p$ which is not stable is said to be unstable. 
Definition 8. A point $z$ is said to be a limit point of $O\left(x_{0}\right)$ if there exists a subsequence $\left(x_{n_{k}}\right)_{k=0,1,2, \ldots}$ of $O\left(x_{0}\right)$ such that $\lim _{k \rightarrow+\infty}\left|x_{n_{k}}-z\right|=0$. The limit set $L\left(x_{0}\right)$ of the orbit $O\left(x_{0}\right)$ is a set of all limit points of the orbit.

Definition 9. An orbit $O\left(x_{0}\right)$ is said to be asymptotically periodic if its limit set is a periodic orbit. An orbit $O\left(x_{0}\right)$ such that $x_{n+p}=x_{n}$ for some $n \geq 1$ and some $p \geq 2$ is said to be eventually periodic.

Next theorems [9] are an important tool for the analysis of the stability.

Theorem 1. Let $x_{s}$ be a stationary state of the difference equation (2.1), where $f$ is continuously differentiable at $x_{s}$. Then the following statements hold:

1. If $\left|f^{\prime}\left(x_{s}\right)\right|<1$, then $x_{s}$ is an asymptotically stable point,

2. If $\left|f^{\prime}\left(x_{s}\right)\right|>1$, then $x_{s}$ is unstable.

Theorem 2. Let $O\left(x_{0}\right)$ be a periodic orbit of period $p$ of the difference equation (2.1), where $f$ is continuously differentiable at all points of orbit. Then the following statements hold:

1. If $\left|f^{\prime}\left(x_{0}\right) \cdot f^{\prime}\left(x_{1}\right) \cdots \cdots f^{\prime}\left(x_{p-1}\right)\right|<1$, then the orbit $O\left(x_{0}\right)$ is stable,

2. If $\left|f^{\prime}\left(x_{0}\right) \cdot f^{\prime}\left(x_{1}\right) \cdots \cdot f^{\prime}\left(x_{p-1}\right)\right|>1$, then the orbit $O\left(x_{0}\right)$ is unstable.

\section{Results}

We consider a model

$$
x_{n+1}=\beta x_{n}-g\left(x_{n}\right)
$$

with a signal function in the following form

$$
g(x)= \begin{cases}-a, & x \leq-\alpha \\ 0, & -\alpha<x<\alpha, \quad(a>0 \text { and } \alpha>0) \\ a, & \alpha \leq x\end{cases}
$$

where $-\alpha, \alpha$ are two thresholds (see Figure 1 ).

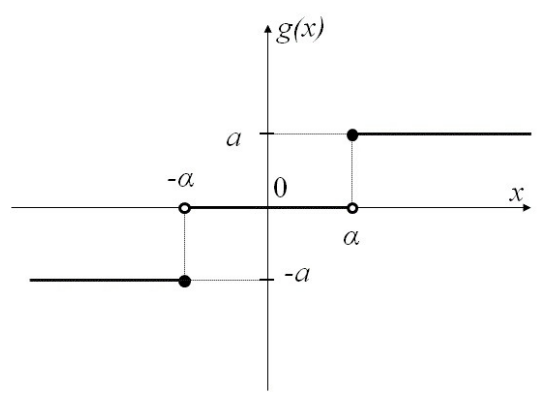

Figure 1. Step signal function $g(x)$.

Further we analyze model (3.1) with a signal function (3.2) depending on the internal decay rate $\beta$. We consider three different situations: $\beta=1,0<\beta<1$ and $\beta>1$. 


\subsection{Model with $\beta=1$}

In this section we consider a model $x_{n+1}=x_{n}-g\left(x_{n}\right)$, when the parameter $\beta=1$.

Theorem 3. Assume that $\beta=1$. Then the following statements hold:

1) If $a<2 \alpha$ then every positive point $x_{0}$ is a stationary state or an eventually stationary state;

2) Every $x_{0} \in\left[0, \alpha\left[\right.\right.$ is a stationary state, i.e., $O\left(x_{0}\right)=\left\{x_{0}, x_{0}, \ldots\right\}$;

3) If $x_{0}=\alpha$ and $a \geq 2 \alpha$ then the orbit $O\left(x_{0}\right)$ is a periodic orbit of period 2, i.e., $O\left(x_{0}\right)=\left\{x_{0}, x_{0}-a, x_{0}, x_{0}-a, \ldots\right\}$;

4) If there exists a positive integer $k$ such that $x_{0}=k a>\alpha$ and

a) $a \geq \alpha$ then the point $x_{0}$ is an eventually stationary state, i.e.,

$$
O\left(x_{0}\right)=\{k a,(k-1) a, \ldots, 2 a, a, 0,0, \ldots\}
$$

b) a natural number $m$ such that $m a<\alpha$ and $(m+1) a \geq \alpha$ then the point $x_{0}$ is an eventually stationary state, i.e.,

$$
O\left(x_{0}\right)=\{k a,(k-1) a, \ldots,(m+1) a, m a, m a, \ldots\} ;
$$

5) If there exists a positive integer $k$ and a real number $\left.k_{1} \in\right]-\alpha, 0[\cup] 0, \alpha[$ such that $x_{0}=k a+k_{1} \geq \alpha$ and $\exists m \in \mathbb{N} \cup\{0\}$ such that $-\alpha<m a+k_{1}<\alpha$ and $(m+1) a \geq \alpha$, then the point $x_{0}$ is an eventually stationary state, i.e.,

$$
O\left(x_{0}\right)=\left\{k a+k_{1},(k-1) a+k_{1}, \ldots,(m+1) a+k_{1}, m a+k_{1}, m a+k_{1}, \ldots\right\} ;
$$

6) For all other positive $x_{0}$ the orbit $O\left(x_{0}\right)$ is periodic of period 2 or eventually periodic of period 2.

Proof. The first five statements are obvious. We prove only Statement 6 ). It is clear that $a>2 \alpha$ and $x_{0}>\alpha$. Then $g\left(x_{0}\right)=a$ and $x_{1}=x_{0}-a$.

Two cases are possible:

Case 1. If $x_{1}=x_{0}-a \leq-\alpha$ then $g\left(x_{1}\right)=-a$ and

$$
x_{2}=x_{1}+a=x_{0}-a+a=x_{0}>\alpha .
$$

Thus we have obtained a periodic orbit of period 2:

$$
O\left(x_{0}\right)=\left\{x_{0}, x_{0}-a, x_{0}, x_{0}-a, \ldots\right\} .
$$

Case 2. If $x_{1}=x_{0}-a \geq \alpha$ then $g\left(x_{1}\right)=a$ and $x_{2}=x_{1}-a=x_{0}-2 a$. Two cases are possible:

Case 2.1. If $x_{2}=x_{0}-2 a \leq-\alpha$ then $g\left(x_{2}\right)=-a$ and $x_{3}=x_{2}+a=x_{0}-a \geq \alpha$. We have obtained an eventually periodic orbit of period 2 :

$$
O\left(x_{0}\right)=\left\{x_{0}, x_{0}-a, x_{0}-2 a, x_{0}-a, x_{0}-2 a, \ldots\right\} .
$$


Case 2.2. If $x_{2}=x_{0}-2 a \geq \alpha$ then $g\left(x_{2}\right)=a$ and $x_{3}=x_{2}-a=x_{0}-3 a$. Two cases are possible. Generally, since $x_{0}>\alpha$ is fixed there exists an integer $k$ such that $x_{k}=x_{0}-k a \leq-\alpha, g\left(x_{k}\right)=-a$ and $x_{k+1}=x_{k}+a=x_{0}-(k-1) a=$ $x_{k-1} \geq \alpha$, i.e., the orbit $O\left(x_{0}\right)$ is an eventually periodic orbit of period 2 :

$O\left(x_{0}\right)=\left\{x_{0}, x_{0}-a, \ldots, x_{0}-(k-1) a, x_{0}-k a, x_{0}-(k-1) a, x_{0}-k a, \ldots\right\}$.

Corollary 1. If $x_{0}$ is negative and in the symmetric form as in Theorem 3 , then conclusions are the same as in Theorem 3 (orbits are symmetric according to 0 ).

\subsection{Model with $0<\beta<1$}

The situation with $0<\beta<1$ is more complicated. We begin with the study of the stability of the stationary state 0 and periodic orbits.

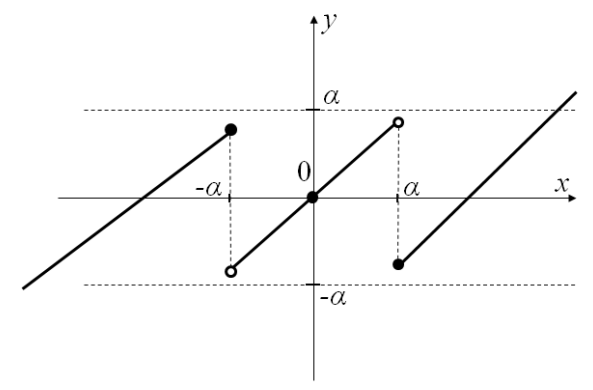

Figure 2. Illustration of Theorem 4: example of a graph of function $h(x)=\beta x-g(x)$, if $0<\beta<1$.

Let $h(x)=\beta x-g(x)$.

Theorem 4. Assume that $0<\beta<1$ (see Figure 2). Then the point 0 is an asymptotically stable stationary state.

Proof. In this case $\delta=\min \{\varepsilon, \alpha\}$ and

$$
\left.\forall x_{0} \in\right]-\delta, \delta\left[: \quad \lim _{n \rightarrow \infty} h^{n}\left(x_{0}\right)=\lim _{n \rightarrow \infty} \beta^{n} x_{0}=0 .\right.
$$

Theorem 5. Assume that $0<\beta<1$ and $\frac{a}{\beta+1}>\alpha$. Then the periodic orbit $O\left(\frac{a}{\beta+1}\right)$ is a stable periodic orbit of period 2 .

Proof. Since $\frac{a}{\beta+1} \geq \alpha$ then $g\left(\frac{a}{\beta+1}\right)=a$ and

$$
h\left(\frac{a}{\beta+1}\right)=\frac{\beta a}{\beta+1}-a=\frac{\beta a-\beta a-a}{\beta+1}=\frac{-a}{\beta+1} .
$$

Since $\frac{-a}{\beta+1} \leq-\alpha$ then $g\left(\frac{-a}{\beta+1}\right)=-a$ and

$$
h\left(\frac{-a}{\beta+1}\right)=\frac{-\beta a}{\beta+1}+a=\frac{-\beta a+\beta a+a}{\beta+1}=\frac{a}{\beta+1} .
$$




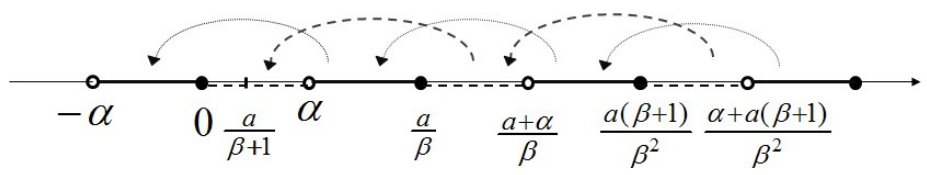

Figure 3. Illustration of Theorem 6 Statement 2: if $x_{0}$ belongs to dotted intervals, then $\exists n_{1} \quad x_{n_{1}} \in\left[0 ; \alpha\left[\right.\right.$; if $x_{0}$ belongs to bold intervals, then $\left.\left.\exists n_{2} \quad x_{n_{2}} \in\right]-\alpha, 0\right]$.

This implies that $O\left(\frac{a}{\beta+1}\right)=\left\{\frac{a}{\beta+1}, \frac{-a}{\beta+1}, \ldots\right\}$ is a periodic orbit of period 2 .

Since $\frac{a}{\beta+1}>\alpha$ then $h(x)=\beta x-g(x)$ is a continuously differentiable function in both points of the orbit and $h^{\prime}\left(\frac{a}{\beta+1}\right)=\beta, h^{\prime}\left(\frac{-a}{\beta+1}\right)=\beta$. Since $\beta<1$ then by Theorem $1 O\left(\frac{a}{\beta+1}\right)$ is a stable periodic orbit of period 2 .

Theorem 6. Assume that $0<\beta<1$. Then the following statements hold:

1) If $\left.x_{0} \in\right] 0, \alpha\left[\right.$ then the orbit $O\left(x_{0}\right)$ is an asymptotically stationary state to 0 , i.e., $\lim _{n \rightarrow \infty} x_{n}=0$ and $O\left(x_{0}\right)=\left\{x_{0}, \beta x_{0}, \beta^{2} x_{0}, \ldots, \beta^{n} x_{0}, \ldots\right\}$.

2) If $\frac{a}{\beta+1}<\alpha$ then the orbit $O\left(x_{0}\right)$ is an asymptotically stationary state to 0 or an eventually stationary state to 0 (see Figure 3).

3) If $\frac{a}{\beta+1}=\alpha$ and

a) $x_{0} \in \Omega_{0}=\left\{\frac{a}{\beta}, \frac{a(\beta+1)}{\beta^{2}}, \ldots, \frac{a \sum_{s=0}^{n} \beta^{s}}{\beta^{n+1}}, \ldots \mid n=0,1,2, \ldots\right\}$ then the orbit $O\left(x_{0}\right)$ is an eventually stationary state to 0 ;

b) $x_{0} \in \Omega_{p 2}=\left\{\frac{\alpha+a}{\beta}, \frac{\alpha+a(\beta+1)}{\beta^{2}}, \ldots, \frac{\alpha+a \sum_{s=0}^{n} \beta^{s}}{\beta^{n+1}}, \ldots \mid n=0,1,2, \ldots\right\}$ then the orbit $O\left(x_{0}\right)$ is an eventually periodic orbit of period 2 :

$$
\left\{x_{0}, x_{1}, \ldots, x_{k}, \alpha,-\alpha, \alpha,-\alpha, \ldots\right\} \text { or }\left\{x_{0}, x_{1}, \ldots, x_{k},-\alpha, \alpha,-\alpha, \alpha, \ldots\right\}
$$

c) $x_{0}=\alpha$ then the orbit $O\left(x_{0}\right)$ is a periodic orbit of period 2 :

$$
\{\alpha,-\alpha, \alpha,-\alpha, \ldots\}
$$

d) If $a)-c)$ are not fulfilled then for positive $x_{0}$ the orbit $O\left(x_{0}\right)$ is an asymptotically stationary state to 0 .

4) If $\frac{a}{\beta+1}>\alpha$ (see Figure 4) and

a) $\left.x_{0} \in\right] 0, \alpha\left[\right.$ then the orbit $O\left(x_{0}\right)$ is an asymptotically stationary state to 0 ;

b) $x_{0} \in \Omega_{0}=\left\{\frac{a}{\beta}, \frac{a(\beta+1)}{\beta^{2}}, \ldots, \frac{a \sum_{s=0}^{n} \beta^{s}}{\beta^{n+1}}, \ldots \mid n=0,1,2, \ldots\right\}$ then the orbit $O\left(x_{0}\right)$ is an eventually stationary state to 0 ;

c) $x_{0} \in \Omega_{p 2}=\left\{\frac{a}{\beta+1}, \frac{a(\beta+2)}{\beta(\beta+1)}, \frac{a\left(\beta^{2}+2 \beta+2\right)}{\beta^{2}(\beta+1)}, \ldots, \frac{a\left(\beta^{n+1}+2 \sum_{s=0}^{n} \beta^{s}\right)}{\beta^{n+1}(\beta+1)}, \ldots \mid n=0,1\right.$, $2, \ldots\}$ then the orbit $O\left(x_{0}\right)$ is an eventually periodic of period 2 ;

d) $\left.x_{0} \in \bigcup_{k=0}^{\infty}\right] \frac{-\alpha+a \sum_{s=0}^{k} \beta^{s}}{\beta^{k+1}}, \frac{\alpha+a \sum_{s=0}^{k} \beta^{s}}{\beta^{k+1}}\left[\backslash \Omega_{0}\right.$ then the orbit $O\left(x_{0}\right)$ is an asymptotically stationary state to 0 ; 


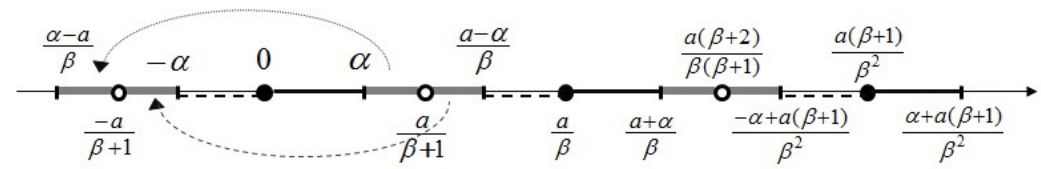

Figure 4. Illustration of Theorem 6 Statement 4: according to the choice of $x_{0}$ the orbit $O\left(x_{0}\right)$ may be asymptotically stationary state 0 or eventually stationary state 0 or eventually periodic of period 2 or asymptotically periodic of period 2 .

e) $x_{0} \in\left[\alpha, \frac{-\alpha+a}{\beta}\right] \cup \bigcup_{k=0}^{\infty}\left[\frac{\alpha+a \sum_{s=0}^{k} \beta^{s}}{\beta^{k+1}}, \frac{-\alpha+a \sum_{s=0}^{k+1} \beta^{s}}{\beta^{k+2}}\right] \backslash \Omega_{p 2}$ then the orbit $O\left(x_{0}\right)$ is asymptotically periodic with the limit set $L\left(x_{0}\right)=\left\{\frac{a}{\beta+1}, \frac{-a}{\beta+1}\right\}$.

6) Situation with negative $x_{0}$ is similar.

\section{Proof.}

Statement 1. If $\left.x_{0} \in\right] 0, \alpha\left[\right.$ then $g\left(x_{0}\right)=0$ and $\left.x_{1}=\beta x_{0} \in\right] 0, \alpha[$. Generally, $x_{n}=\beta^{n} x_{0}$ for all $n \geq 0$. Thus $O\left(x_{0}\right)=\left\{x_{0}, \beta x_{0}, \beta^{2} x_{0}, \ldots, \beta^{n} x_{0}, \ldots\right\}$.

Since $0<\beta<1$, we have $\lim _{n \rightarrow \infty} x_{n}=\lim _{n \rightarrow \infty} \beta^{n} x_{0}=0$.

\section{Statement 2 .}

Case 1. Assume $\frac{a}{\beta}>\alpha$. If $x_{0} \in \Omega_{0}=\left\{\frac{a}{\beta}, \frac{a(\beta+1)}{\beta^{2}}, \ldots, \frac{a \sum_{s=0}^{n} \beta^{s}}{\beta^{n+1}}, \ldots \mid n=\right.$ $0,1,2, \ldots\}$ then $O\left(x_{0}\right)=\left\{x_{0}, x_{1}, \ldots, x_{k}, 0,0, \ldots\right\}$, i.e., the orbit $O\left(x_{0}\right)$ is an eventually stationary state to 0 .

If $\left.x_{0} \in\right] \alpha, a / \beta[$ then

$$
-\alpha<\frac{-a}{\beta+1}=\frac{a \beta}{\beta+1}-a<\beta \alpha-a<x_{1}=\beta x_{0}-a<\beta \frac{a}{\beta}-a=0 .
$$

Since $-\alpha<x_{1}<0$, then $x_{n}=\beta^{n-1}\left(\beta x_{0}-a\right)$ for all $n \geq 2$ and the orbit $O\left(x_{0}\right)$ is an asymptotically stationary state to 0 .

$$
\begin{aligned}
& \text { If } \left.x_{0} \in\right] \frac{\alpha+a \sum_{s=0}^{k} \beta^{s}}{\beta^{k+1}}, \frac{a \sum_{s=0}^{k+1} \beta^{s}}{\beta^{k+2}}\left[\text { then } x_{1}=\beta x_{0}-a\right. \text { : } \\
& x_{1}>\beta \frac{\alpha+a \sum_{s=0}^{k} \beta^{s}}{\beta^{k+1}}-a=\frac{\alpha+a \sum_{s=0}^{k} \beta^{s}-a \beta^{k}}{\beta^{k}}=\frac{\alpha+a \sum_{s=0}^{k-1} \beta^{s}}{\beta^{k}}, \\
& x_{1}<\frac{\beta a \sum_{s=0}^{k+1} \beta^{s}}{\beta^{k+2}}-a=\frac{a \sum_{s=0}^{k+1} \beta^{s}-a \beta^{k}}{\beta^{k+1}}=\frac{a \sum_{s=0}^{k} \beta^{s}}{\beta^{k+1}},
\end{aligned}
$$

that is, if $x_{0}$ belongs to the $k$-th interval, then $x_{1}$ belongs to the $(k-1)$-th interval, $x_{2}$ belongs to the $(k-2)$-th interval, $\left.\ldots, x_{k} \in\right] \alpha, \frac{a}{\beta}[$. Therefore in this case the orbit $O\left(x_{0}\right)$ is an asymptotically stationary state to 0 .

Proof for $\left.x_{0} \in\right] \frac{a}{\beta}, \frac{a+\alpha}{\beta}\left[\right.$ and $\left.x_{0} \in\right] \frac{a \sum_{s=0}^{k} \beta^{s}}{\beta^{k+1}}, \frac{\alpha+a \sum_{s=0}^{k} \beta^{s}}{\beta^{k+1}}[$ is similar.

Case 2. If $\frac{a \sum_{s=0}^{k} \beta^{s}}{\beta^{k+1}}>\alpha$ for $k=1,2,3, \ldots$ then the situation and proof is similar as in Case 1.

Case 3. Assume $\frac{a}{\beta} \leq \alpha$. Let $x_{0} \geq \alpha$. Then $x_{1}=\beta x_{0}-a \geq \frac{\beta a}{\beta}-a=0$. Since $x_{0}$ is fixed, then exists an integer $k$ such that $x_{k-1} \geq \alpha$ and $0 \leq x_{k}<\alpha$. Thus we have obtained that the orbit $O\left(x_{0}\right)$ is an asymptotically stationary state to 0 . 
Statement 3. Statements a) - c) are obvious. We prove Statement d) for positive $x_{0}$. Let $\frac{a}{\beta+1}=\alpha<x_{0}<\frac{a}{\beta}$. Then

$$
-\alpha=\frac{-a}{\beta+1}=\frac{a \beta}{\beta+1}-a<x_{1}=\beta x_{0}-a<\frac{a \beta}{\beta}-a=0 .
$$

Thus

$$
\begin{aligned}
& x_{2}=\beta x_{1}=\beta\left(\beta x_{0}-a\right), \quad-\alpha<x_{2}<0, \\
& \ldots \\
& x_{n}=\beta x_{n-1}=\beta^{n-1}\left(\beta x_{0}-a\right), \quad-\alpha<x_{n}<0,
\end{aligned}
$$

Since $0<\beta<1$, we have $\lim _{n \rightarrow \infty} x_{n}=\lim _{n \rightarrow \infty} \beta^{n} x_{0}=0$.

If $\left.x_{0} \in\right] \frac{\alpha+a \sum_{s=0}^{k} \beta^{s}}{\beta^{k+1}}, \frac{a \sum_{s=0}^{k+1} \beta^{s}}{\beta^{k+2}}\left[\right.$ then $x_{1}=\beta x_{0}-a$ :

$$
\begin{aligned}
& x_{1}>\beta \frac{\alpha+a \sum_{s=0}^{k} \beta^{s}}{\beta^{k+1}}-a=\frac{\alpha+a \sum_{s=0}^{k} \beta^{s}-a \beta^{k}}{\beta^{k}}=\frac{\alpha+a \sum_{s=0}^{k-1} \beta^{s}}{\beta^{k}}, \\
& x_{1}<\frac{\beta a \sum_{s=0}^{k+1} \beta^{s}}{\beta^{k+2}}-a=\frac{a \sum_{s=0}^{k+1} \beta^{s}-a \beta^{k}}{\beta^{k+1}}=\frac{a \sum_{s=0}^{k} \beta^{s}}{\beta^{k+1}},
\end{aligned}
$$

that is, if $x_{0}$ belongs to the $k$-th interval, then $x_{1}$ belongs to the $(k-1)$-th interval, $x_{2}$ belongs to the $(k-2)$-th interval, $\left.\ldots, x_{k} \in\right] \alpha, \frac{a}{\beta}[$. Therefore in this case the orbit $O\left(x_{0}\right)$ is an asymptotically stationary state to 0 .

Proof for $\left.x_{0} \in\right] \frac{a}{\beta}, \frac{a+\alpha}{\beta}\left[\right.$ and $\left.x_{0} \in\right] \frac{a \sum_{s=0}^{k} \beta^{s}}{\beta^{k+1}}, \frac{\alpha+a \sum_{s=0}^{k} \beta^{s}}{\beta^{k+1}}[$ is similar.

Statement 4. We prove only Statement e) for $x_{0} \in\left[\alpha, \frac{-\alpha+a}{\beta}\right]$. Proofs of the statements a)-d) and the rest of the values $x_{0}$ are similar as for Statement 3 ) and 4). Assume $\alpha \leq x_{0} \leq \frac{-\alpha+a}{\beta}$. Then

$$
\frac{\alpha-a}{\beta}<\alpha \beta-a \leq x_{1}=\beta x_{0}-a \leq \beta \frac{-\alpha+a}{\beta}-a=-\alpha .
$$

We show that the inequality $\frac{\alpha-a}{\beta}<\alpha \beta-a$ holds by making equivalent transformations:

$$
\begin{aligned}
& \frac{\alpha}{\beta}-\frac{a}{\beta}-\alpha \beta+a<0, \quad-\frac{\alpha}{\beta}+\frac{a}{\beta}+\alpha \beta-a>0 \\
& -\alpha\left(\frac{1}{\beta}-\beta\right)+a\left(\frac{1}{\beta}-\beta\right)>0, \quad(a-\alpha)\left(\frac{1}{\beta}-\beta\right)>0 .
\end{aligned}
$$

Last inequality holds as both multipliers are positive, that is, since $0<\beta<1$ then $\frac{1}{\beta}-\beta>0$ and $a>\frac{a}{\beta+1}>\alpha$.

Since $\frac{\alpha-a}{\beta}<x_{1} \leq-\alpha$ then

$$
\begin{aligned}
x_{2} & =\beta x_{1}+a=\beta\left(\beta x_{0}-a\right)+a=\beta^{2} x_{0}+a(1-\beta)=\beta^{2} x_{0}+\frac{a\left(1-\beta^{2}\right)}{1+\beta} \\
& =\beta^{2}\left(x_{0}-\frac{a}{\beta+1}\right)+\frac{a}{1+\beta} .
\end{aligned}
$$


But on the other hand

$$
\alpha=\frac{\beta(\alpha-a)}{\beta}+a<x_{2}=\beta x_{1}+a \leq \beta(-\alpha)+a<\frac{a-\alpha}{\beta} .
$$

Generally, we have

$$
\begin{aligned}
& \alpha<x_{2 n}=\beta^{2 n}\left(x_{0}-\frac{a}{\beta+1}\right)+\frac{a}{\beta+1}<\frac{a-\alpha}{\beta}, \quad n=1,2, \ldots, \\
& \frac{\alpha-a}{\beta}<x_{2 n+1}=\beta^{2 n+1}\left(x_{0}-\frac{a}{\beta+1}\right)-\frac{a}{\beta+1}<-\alpha, \quad n=0,1,2, \ldots
\end{aligned}
$$

Therefore $\lim _{n \rightarrow \infty} x_{2 n}=\frac{a}{\beta+1}$ and $\lim _{n \rightarrow \infty} x_{2 n+1}=\frac{-a}{\beta+1}$. Thus we have proved that $L\left(x_{0}\right)=\left\{\frac{a}{\beta+1}, \frac{-a}{\beta+1}\right\}$.

\subsection{Model with $\beta>1$}

In this section we consider a situation with $\beta>1$ and obtain periodic orbits of period 2, 4 and others for difference equation (3.1). This case is very interesting while it is possible to find a periodic orbit of an arbitrary chosen period.

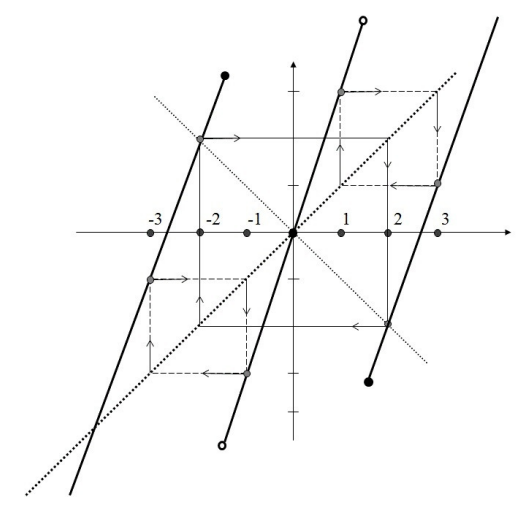

Figure 5. If $\beta=3, a=8$ and $\alpha=1.5$, then we have three periodic orbits of period 2: $\{-1,-3\},\{1,3\}$ and $\{2,-2\}$.

At first we notice that a periodic orbit of period 2 depends on parameters. Four cases are possible:

1) If $\frac{a}{\beta+1} \geq \alpha$ and $\frac{a}{\beta^{2}-1}<\alpha$, then there exists three periodic orbits of period 2, i.e., $\left\{-\frac{a}{\beta^{2}-1},-\frac{a \beta}{\beta^{2}-1}\right\},\left\{\frac{a}{\beta^{2}-1}, \frac{a \beta}{\beta^{2}-1}\right\}$ and $\left\{\frac{a}{\beta+1},-\frac{a}{\beta+1}\right\}$. We have an arrangement

$$
-\frac{a \beta}{\beta^{2}-1}<-\frac{a}{\beta+1} \leq-\alpha<-\frac{a}{\beta^{2}-1}<0<\frac{a}{\beta^{2}-1}<\alpha \leq \frac{a}{\beta+1}<\frac{a \beta}{\beta^{2}-1} .
$$

For example, if $\beta=3, a=8$ and $\alpha=1.5$, then we have three periodic orbits of period 2: $\{-1,-3\},\{1,3\}$ and $\{2,-2\}$, see Figure 5 .

2) If $\frac{a}{\beta+1} \geq \alpha$ and $\frac{a}{\beta^{2}-1} \geq \alpha$, then there exists one periodic orbit of period 2 , which is $\left\{\frac{a}{\beta+1},-\frac{a}{\beta+1}\right\}$. 
3) If $\frac{a}{\beta^{2}-1}<\alpha \leq \frac{a \beta}{\beta^{2}-1}$ and $\frac{a}{\beta+1}<\alpha$, then there exists two periodic orbits of period 2 , that are, $\left\{-\frac{a}{\beta^{2}-1},-\frac{a \beta}{\beta^{2}-1}\right\}$ and $\left\{\frac{a}{\beta^{2}-1}, \frac{a \beta}{\beta^{2}-1}\right\}$.

4) If $\frac{a \beta}{\beta^{2}-1}<\alpha$, there are no periodic orbits of period 2 .

For the periodic orbit of period 4 more cases are possible. We consider some of them:

1) $\left\{x_{0}=\frac{a\left(\beta^{3}+\beta^{2}+\beta\right)}{\beta^{4}-1}, x_{1}=\frac{a\left(\beta^{3}+\beta^{2}+1\right)}{\beta^{4}-1}, x_{2}=\frac{a\left(\beta^{3}+\beta+1\right)}{\beta^{4}-1}, x_{3}=\frac{a\left(\beta^{2}+\beta+1\right)}{\beta^{4}-1}\right\}$; in this case it is necessary that $x_{3}<\alpha \leq x_{2}<x_{1}<x_{0}$. For example, if $\beta=2$, $a=15, \alpha=8$, then $\{14,13,11,7\}$ is a periodic orbit of period 4 (see Figure 6 ). 2) $\left\{x_{0}=\frac{a\left(\beta^{3}+\beta^{2}\right)}{\beta^{4}-1}, x_{1}=\frac{a\left(\beta^{3}+1\right)}{\beta^{4}-1}, x_{2}=\frac{a(\beta+1)}{\beta^{4}-1}, x_{3}=\frac{a\left(\beta^{2}+\beta\right)}{\beta^{4}-1}\right\}$; in this case it is necessary that $x_{2}<x_{3}<\alpha \leq x_{1}<x_{0}$. For example, with the same parameters as above $\beta=2, a=15, \alpha=8$ there exists a periodic orbit of period 4: $\{12,9,3,6\}$ (see Figure 6).

3) $\left\{x_{0}=\frac{a \beta^{3}}{\beta^{4}-1}, x_{1}=\frac{a}{\beta^{4}-1}, x_{2}=\frac{a \beta}{\beta^{4}-1}, x_{3}=\frac{a \beta^{2}}{\beta^{4}-1}\right\}$; in this case it is necessary that $x_{1}<x_{2}<x_{3}<\alpha \leq x_{0}$. For example, with the same parameters as above $\beta=2, a=15, \alpha=8$ there exists a periodic orbit of period $4:\{8,1,2,4\}$ (see Figure 6).

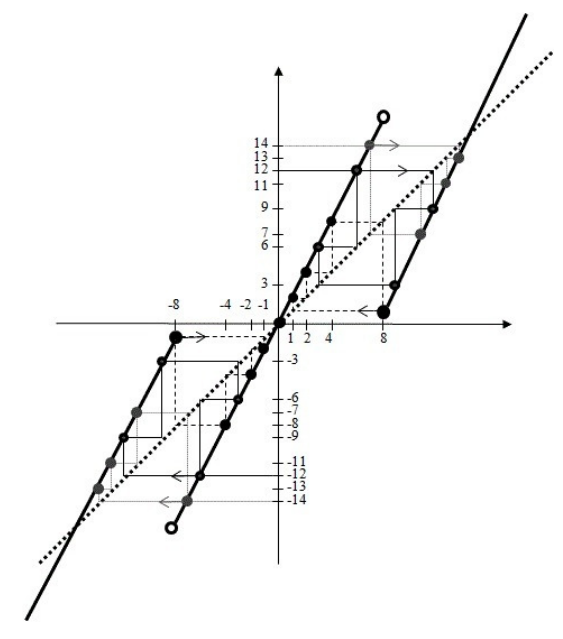

Figure 6. If $\beta=2, a=15, \alpha=8$, then there exists at least three periodic orbit of period $4:\{14,13,11,7\},\{12,9,3,6\}$ and $\{8,1,2,4\}$.

4) another type of the orbit is: if $\alpha<\frac{a(\beta-1)}{\beta^{2}+1}$, then

$$
\left\{\frac{a(\beta+1)}{\beta^{2}+1}, \frac{a(\beta-1)}{\beta^{2}+1},-\frac{a(\beta+1)}{\beta^{2}+1},-\frac{a(\beta-1)}{\beta^{2}+1}\right\}
$$

is a periodic orbit of period 4. For example, $\beta=2, a=15$ and $\alpha=2$ forms a periodic orbit $\{9,3,-9,-3\}$ (see Figure 7 a)).

5) more interesting orbit is $\left\{x_{0}=\frac{a\left(\beta^{3}-\beta^{2}-1\right)}{\beta^{4}-1}, x_{1}=\frac{a\left(-\beta^{3}-\beta+1\right)}{\beta^{4}-1}, x_{2}=\right.$ $\left.\frac{a\left(-\beta^{2}+\beta-1\right)}{\beta^{4}-1}, x_{3}=\frac{a\left(-\beta^{3}+\beta^{2}-\beta\right)}{\beta^{4}-1}\right\}$. In this case we need $x_{1}<x_{3} \leq-\alpha<x_{2}<$ $0<\alpha \leq x_{0}$. For example, if $\beta=3, a=80, \alpha=10$, then $\{17,-29,-7,-21\}$ is a periodic orbit of period 4 (see Figure $7 \mathrm{~b}$ )). 


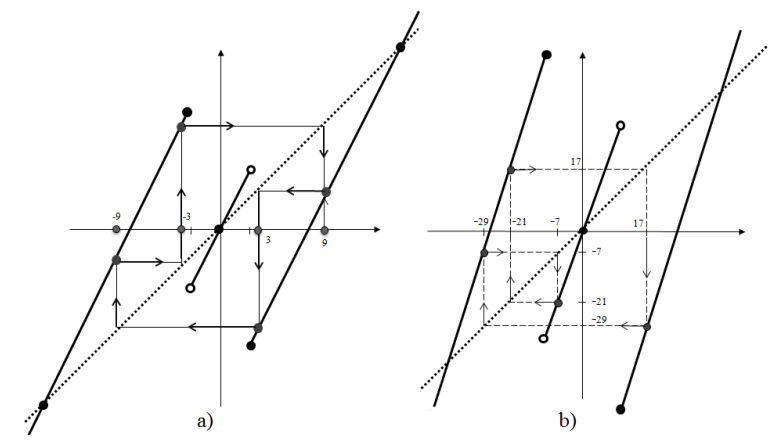

Figure 7. a) If $\beta=2, a=15$ and $\alpha=2$, then there exists periodic orbit with period 4: $\{9,3,-9,-3\}$ and all points of orbit belong to external lines. b) If $\beta=3, a=80$ and $\alpha=10$, then there exists periodic orbit with period $4:\{17,-29,-7,-21\}$.

But even more strong result can be proved: we can construct periodic orbits of an arbitrary period.

Theorem 7. If there exists a positive integer $k \geq 2$ such that

$$
\frac{\beta^{2 k}-2 \beta^{2 k-2}+1}{\beta^{2 k}-1}>\frac{\alpha(\beta+1)}{a},
$$

then difference equation (3.1) has a periodic orbit of period $2 k$ and all periodic orbits are unstable.

Proof. Suppose (3.3) holds. We construct a periodic orbit $O\left(x_{0}\right)$ of period $2 k$ such that

$$
\begin{aligned}
& x_{0}>\alpha, \quad x_{1}>\alpha, \quad x_{2}<-\alpha, \quad x_{3}<-\alpha, \\
& (-1)^{i} x_{i}>\alpha \text { for } i=4, \ldots, 2 k-1, \quad x_{2 k}=x_{0} .
\end{aligned}
$$

Then

$$
\begin{aligned}
& x_{0}>\alpha, \quad x_{1}=\beta x_{0}-a>\alpha \\
& x_{2}=\beta^{2} x_{0}-a \beta-a<-\alpha, \\
& x_{3}=\beta^{3} x_{0}-a \beta^{2}-a \beta+a<-\alpha, \\
& x_{4}=\beta^{4} x_{0}-a \beta^{3}-a \beta^{2}+a \beta+a>\alpha \\
& x_{5}=\beta^{5} x_{0}-a \beta^{4}-a \beta^{3}+a \beta^{2}+a \beta-a<-\alpha, \\
& x_{6}=\beta^{6} x_{0}-a \beta^{5}-a \beta^{4}+a \beta^{3}+a \beta^{2}-a \beta+a>\alpha, \\
& \ldots \quad+\quad \quad+a \beta^{2 k-7}-\cdots+a \beta-a<-\alpha, \\
& x_{2 k-1}=\beta^{2 k-1} x_{0}-a \beta^{2 k-2}-a \beta^{2 k-3}+a \beta^{2 k-4}+a \beta^{2 k-5}-a \beta^{2 k-6} \\
& \quad+a \beta^{2 k-6}-a \beta^{2 k-7}+\cdots+a \beta^{2}-a \beta+a=x_{0}>\alpha . \\
& x_{2 k}=\beta^{2 k} x_{0}-a \beta^{2 k-1}-a \beta^{2 k-2}+a \beta^{2 k-3}+a \beta^{2 k-4}-a \beta^{2 k-5}
\end{aligned}
$$


Since $x_{2 k}=x_{0}$, then

$$
x_{0}=\frac{a\left(\beta^{2 k-1}+\beta^{2 k-2}-\beta^{2 k-3}-\beta^{2 k-4}+\beta^{2 k-5}-\beta^{2 k-6}+\cdots-\beta^{2}+\beta-1\right)}{\beta^{2 k}-1},
$$

therefore

$$
x_{0}=\frac{a\left(\beta^{2 k}+2 \beta^{2 k-1}-2 \beta^{2 k-3}-1\right)}{\left(\beta^{2 k}-1\right)(\beta+1)} .
$$

Since (3.3) holds, then $x_{0}>\alpha$. Then

$$
\begin{aligned}
x_{1} & =\beta x_{0}-a=\frac{a\left(\beta^{2 k+1}+2 \beta^{2 k}-2 \beta^{2 k-2}-\beta\right)}{\left(\beta^{2 k}-1\right)(\beta+1)}-a \\
& =\frac{a\left(\beta^{2 k+1}+2 \beta^{2 k}-2 \beta^{2 k-2}-\beta-\beta^{2 k+1}-\beta^{2 k}+\beta+1\right)}{\left(\beta^{2 k}-1\right)(\beta+1)} \\
& =\frac{a\left(\beta^{2 k}-2 \beta^{2 k-2}+1\right)}{\left(\beta^{2 k}-1\right)(\beta+1)}>\alpha .
\end{aligned}
$$

We show that

$$
\min \left\{x_{0}, x_{1}, x_{4}, x_{6}, \ldots, x_{2 k-2}\right\}=x_{1}>\alpha
$$

At first we notice that

$$
x_{1}=\frac{a\left(\beta^{2 k}-2 \beta^{2 k-2}+1\right)}{\left(\beta^{2 k}-1\right)(\beta+1)}<x_{0}=\frac{a\left(\beta^{2 k}+2 \beta^{2 k-1}-2 \beta^{2 k-3}-1\right)}{\left(\beta^{2 k}-1\right)(\beta+1)}
$$

because for all $k=2,3, \ldots$ and $\beta>1$

$$
\beta^{2 k-1}-\beta^{2 k-3}+\beta^{2 k-2}-1>0 .
$$

Further we show that $x_{1}<x_{4}$. We notice that $x_{4}=\beta^{3} x_{1}-\beta^{2} a+\beta a+a$. Then

$$
\begin{aligned}
x_{1}<x_{4}= & \beta^{3} x_{1}-\beta^{2} a+\beta a+a \\
\Leftrightarrow \quad 0< & \left(\beta^{3}-1\right) x_{1}-\beta^{2} a+\beta a+a \\
\Leftrightarrow \quad 0< & \frac{a\left(\beta^{2 k}-2 \beta^{2 k-2}+1\right)\left(\beta^{3}-1\right)}{\left(\beta^{2 k}-1\right)(\beta+1)}-\beta^{2} a+\beta a+a \\
\Leftrightarrow \quad 0< & \beta^{2 k+3}-2 \beta^{2 k+1}+\beta^{3}-\beta^{2 k}+2 \beta^{2 k-2}-1-\beta^{2 k+3}+\beta^{2 k+2} \\
& \quad+\beta^{2 k+1}-\beta^{2 k+2}+\beta^{2 k+1}+\beta^{2 k}+\beta^{3}-\beta^{2}-\beta+\beta^{2}-\beta-1 \\
= & 2 \beta^{2 k-2}+2 \beta^{3}-2 \beta-2=2 \beta\left(\beta^{2 k-3}-1\right)+2\left(\beta^{3}-1\right)
\end{aligned}
$$

because $\beta>1$ and $k \geq 2$. Finally we show that $x_{2 m-2}<x_{2 m}, 3 \leq m<k$. 
Indeed

$$
\begin{aligned}
x_{2 m-2}= & \beta^{2 m-2} x_{0}-a \beta^{2 m-3}-a \beta^{2 m-4}+a \beta^{2 m-5}+a \beta^{2 m-6}-a \beta^{2 m-7} \\
& +a \beta^{2 m-8}-\cdots-a \beta+a \\
< & x_{2 m}=\beta^{2 m} x_{0}-a \beta^{2 m-1}-a \beta^{2 m-2}+a \beta^{2 m-3}+a \beta^{2 m-4}-a \beta^{2 m-5} \\
& +a \beta^{2 m-6}-\cdots-a \beta+a \\
\Leftrightarrow \quad 0< & \left(\beta^{2 m}-\beta^{2 m-2}\right) x_{0}-a \beta^{2 m-1}-a \beta^{2 m-2}+2 a \beta^{2 m-3} \\
& \quad+2 a \beta^{2 m-4}-2 a \beta^{2 m-5} \\
\Leftrightarrow \quad 0< & \frac{\beta^{2 m-2}\left(\beta^{2}-1\right) a\left(\beta^{2 k}+2 \beta^{2 k-1}-2 \beta^{2 k-3}-1\right)}{\left(\beta^{2 k}-1\right)(\beta+1)} \\
& \quad-a \beta^{2 m-1}-a \beta^{2 m-2}+2 a \beta^{2 m-3}+2 a \beta^{2 m-4}-2 a \beta^{2 m-5} \\
= & \frac{2 a \beta^{2 m-5}}{\beta^{2 k}-1}\left(\beta^{3}-\beta^{2}-\beta+1\right)=\frac{2 a \beta^{2 m-5}}{\beta^{2 k}-1}(\beta-1)\left(\beta^{2}-1\right) .
\end{aligned}
$$

Since $\beta>1$ is greater than 0 therefore

$$
\frac{2 a \beta^{2 m-5}}{\beta^{2 k}-1}(\beta-1)\left(\beta^{2}-1\right)>0 .
$$

We show that

$$
x_{2}<-\alpha \text { and } \max \left\{x_{3}, x_{5}, x_{7}, \ldots, x_{2 k-1}\right\}=x_{2 k-1}<-\alpha .
$$

Since (3.3) holds, then $\frac{a\left(2 \beta^{2 k-2}-\beta^{2 k}-1\right)}{\left(\beta^{2 k}-1\right)(\beta+1)}<-\alpha$. At first we show that $x_{2}<-\alpha$ :

$$
\begin{aligned}
x_{2} & =\beta^{2} x_{0}-a \beta-a=\frac{\beta^{2} a\left(\beta^{2 k}+2 \beta^{2 k-1}-2 \beta^{2 k-3}-1\right)}{\left(\beta^{2 k}-1\right)(\beta+1)}-a(\beta+1) \\
& =\frac{a\left(-\beta^{2 k}-2 \beta^{2 k-1}+2 \beta+1\right)}{\left(\beta^{2 k}-1\right)(\beta+1)} \\
& =\frac{a\left(2 \beta^{2 k-2}-\beta^{2 k}-1-2 \beta^{2 k-2}-2 \beta^{2 k-1}+2 \beta+2\right)}{\left(\beta^{2 k}-1\right)(\beta+1)} \\
& <-\alpha-\frac{2 a\left(\beta^{2 k-2}+\beta^{2 k-1}-\beta-1\right)}{\left(\beta^{2 k}-1\right)(\beta+1)}<-\alpha .
\end{aligned}
$$

By construction $x_{2 k-1}=\frac{x_{0}-a}{\beta}$, then

$$
\begin{aligned}
x_{2 k-1} & =\frac{a\left(\beta^{2 k}+2 \beta^{2 k-1}-2 \beta^{2 k-3}-1\right)}{\left(\beta^{2 k}-1\right)(\beta+1) \beta}-\frac{a}{\beta} \\
& =\frac{a\left(2 \beta^{2 k-1}-2 \beta^{2 k-3}-\beta^{2 k+1}\right)}{\left(\beta^{2 k}-1\right)(\beta+1) \beta}=\frac{a\left(2 \beta^{2 k-2}-2 \beta^{2 k-4}-\beta^{2} k\right)}{\left(\beta^{2 k}-1\right)(\beta+1)} \\
& =\frac{a\left(2 \beta^{2 k-2}-\beta^{2 k}-1+1-2 \beta^{2 k-4}\right)}{\left(\beta^{2 k}-1\right)(\beta+1)}<-\alpha-\frac{a\left(2 \beta^{2 k-4}-1\right)}{\left(\beta^{2 k}-1\right)(\beta+1)}<-\alpha,
\end{aligned}
$$

because $\beta>1$ and $k \geq 2$. Similar as (3.7) it is possible to show that $x_{2 m-3}<$ $x_{2 m-1}$ for all $3 \leq m \leq k$. 
If the assumptions of Theorem 7 hold, the orbit $O\left(x_{0}\right)$ where $x_{0}$ is defined by (3.5) (or (3.6)), satisfies (3.4) and is periodic orbit of period $2 k$ for (3.1).

By construction the orbit $O\left(x_{0}\right)$ does not contain $-\alpha$ and $\alpha$ therefore function $h(x)=\beta x-g(x)$ is differentiable in all points of orbit $O\left(x_{0}\right)$. Since $\beta>1$ then by Theorem 2 the orbit $O\left(x_{0}\right)$ is an unstable periodic orbit of period $2 k$.

Theorem 8. If there exists a positive integer $k$ such that

$$
\frac{\beta^{2 k+1}-2 \beta^{2 k-1}-1}{\beta^{2 k+1}-1}>\frac{\alpha(\beta+1)}{a},
$$

then the difference equation (3.1) has a periodic orbit of period $2 k+1$ and all periodic orbits are unstable.

Proof. Suppose (3.8) holds. We will construct a periodic orbit $O\left(x_{0}\right)$ of period $2 k+1$ such that

$$
\begin{gathered}
x_{0}>\alpha, \quad x_{1}<-\alpha, \quad x_{2}<-\alpha \\
(-1)^{i} x_{i}<-\alpha \text { for } i=3, \ldots, 2 k, \quad x_{2 k+1}=x_{0} .
\end{gathered}
$$

Then

$$
\begin{aligned}
& x_{0}>\alpha, \quad x_{1}=\beta x_{0}-a<-\alpha, \\
& x_{2}=\beta^{2} x_{0}-a \beta+a<-\alpha, \\
& x_{3}=\beta^{3} x_{0}-a \beta^{2}+a \beta+a>\alpha, \\
& x_{4}=\beta^{4} x_{0}-a \beta^{3}+a \beta^{2}+a \beta-a<-\alpha, \\
& \ldots \\
& \quad \begin{array}{l}
x_{2 k}=\beta^{2 k} x_{0}-a \beta^{2 k-1}+a \beta^{2 k-2}+a \beta^{2 k-3}-a \beta^{2 k-4}+a \beta^{2 k-5} \\
\quad-a \beta^{2 k-6}+\cdots+a \beta-a<-\alpha, \\
x_{2 k+1}=\beta^{2 k+1} x_{0}-a \beta^{2 k}+a \beta^{2 k-1}+a \beta^{2 k-2}-a \beta^{2 k-3}+a \beta^{2 k-4}
\end{array} \quad \quad-a \beta^{2 k-5}+a \beta^{2 k-6}+\cdots+a \beta^{2}-a \beta+a=x_{0}>\alpha .
\end{aligned}
$$

Since $x_{2 k+1}=x_{0}$, then

$$
x_{0}=\frac{a\left(\beta^{2 k}-\beta^{2 k-1}-\beta^{2 k-2}+\beta^{2 k-3}-\beta^{2 k-4}+\beta^{2 k-5}-\cdots-\beta^{2}+\beta-1\right)}{\beta^{2 k+1}-1},
$$

therefore

$$
x_{0}=\frac{a\left(\beta^{2 k+1}-2 \beta^{2 k-1}-1\right)}{\left(\beta^{2 k+1}-1\right)(\beta+1)} .
$$

Since (3.8) holds, then $x_{0}>\alpha$. We show that

$$
\alpha<x_{0}<x_{2 k-1}<x_{2 k-3}<\cdots<x_{5}<x_{3} .
$$


At first we show that

$$
\begin{aligned}
x_{0}< & x_{2 k-1}=\beta^{2 k-1} x_{0}-a \beta^{2 k-2}+a \beta^{2 k-3}+a \beta^{2 k-4}-a \beta^{2 k-5} \\
& +a \beta^{2 k-6}-\cdots-a \beta+a .
\end{aligned}
$$

Because (3.10) holds we prove that

$$
\begin{aligned}
0< & \frac{\left(\beta^{2 k-1}-1\right) a\left(\beta^{2 k+1}-2 \beta^{2 k-1}-1\right)}{\left(\beta^{2 k+1}-1\right)(\beta+1)}-a \beta^{2 k-2}+a \beta^{2 k-3} \\
& +a \beta^{2 k-4}-\cdots-a \beta+a .
\end{aligned}
$$

The right side of the last inequality by algebraic transformations is equal to

$$
\frac{a\left(2 \beta^{2 k-1}-2 \beta^{2 k-3}\right)}{\left(\beta^{2 k+1}-1\right)(\beta+1)}=\frac{2 a \beta^{2 k-3}(\beta-1)}{\beta^{2 k+1}-1},
$$

which is greater than 0 since $\beta>1$ and $k \geq 2$.

Secondly we show that $x_{2 m-1}<x_{2 m-3}, 3 \leq m<k$. Indeed

$$
\begin{aligned}
\left(x_{2 m-1}=\right. & \beta^{2 m-1} x_{0}-a \beta^{2 m-2}+a \beta^{2 m-3}+a \beta^{2 m-4}-a \beta^{2 m-5}+a \beta^{2 m-6} \\
& -a \beta^{2 m-7}-\cdots-a \beta+a \\
< & x_{2 m-3}=\beta^{2 m-3} x_{0}-a \beta^{2 m-4}+a \beta^{2 m-5}+a \beta^{2 m-6}-a \beta^{2 m-7} \\
& \left.+a \beta^{2 m-8}-a \beta^{2 m-9}-\cdots-a \beta+a\right) \\
\Leftrightarrow \quad 0< & \left(\beta^{2 m-3}-\beta^{2 m-1}\right) x_{0}+a \beta^{2 m-2}-a \beta^{2 m-3}-2 a \beta^{2 m-4}+2 a \beta^{2 m-5} \\
\Leftrightarrow \quad 0< & \frac{\beta^{2 m-3}\left(1-\beta^{2}\right) a\left(\beta^{2 k+1}-2 \beta^{2 k-1}-1\right)}{\left(\beta^{2 k+1}-1\right)(\beta+1)} \\
& \quad+a \beta^{2 m-2}-a \beta^{2 m-3}-2 a \beta^{2 m-4}+2 a \beta^{2 m-5} \\
\Leftrightarrow \quad 0< & \frac{2 a \beta^{2 m-5}(\beta-1)}{\beta^{2 k+1}-1} .
\end{aligned}
$$

Since $\beta>1$ and $m \geq 3$, the last inequality holds.

Now we need to show that

$$
\max \left\{x_{1}, x_{2}, x_{4}, \ldots, x_{2 k}\right\}<-\alpha
$$

In this case it is possible to prove that $x_{1}<x_{2 k}<x_{2 k-2}<\cdots<x_{2}<-\alpha$.

From (3.10) follows that

$$
x_{1}=\beta x_{0}-a=\frac{a \beta\left(\beta^{2 k+1}-2 \beta^{2 k-1}-1\right)}{\left(\beta^{2 k+1}-1\right)(\beta+1)}-a .
$$

By the construction

$$
x_{2 k}=\frac{x_{0}}{\beta}-\frac{a}{\beta}=\frac{a \beta\left(\beta^{2 k+1}-2 \beta^{2 k-1}-1\right)}{\beta\left(\beta^{2 k+1}-1\right)(\beta+1)}-\frac{a}{\beta} .
$$


Inequality $x_{1}<x_{2 k}$ is fulfilled, if the following inequalities holds

$$
\begin{gathered}
\frac{\beta^{2}\left(\beta^{2 k+1}-2 \beta^{2 k-1}-1\right)}{\left(\beta^{2 k+1}-1\right)(\beta+1)}-\beta<\frac{\beta\left(\beta^{2 k+1}-2 \beta^{2 k-1}-1\right)}{\left(\beta^{2 k+1}-1\right)(\beta+1)}-1 \\
\Leftrightarrow \quad \frac{\beta^{2 k+3}-2 \beta^{2 k+1}-\beta^{2}-\beta^{2 k+3}-\beta^{2 k+2}+\beta^{2}+\beta}{\left(\beta^{2 k+1}-1\right)(\beta+1)} \\
\quad<\frac{\beta^{2 k+1}-2 \beta^{2 k-1}-1-\beta^{2 k+2}-\beta^{2 k+1}+\beta+1}{\left(\beta^{2 k+1}-1\right)(\beta+1)} \\
\Leftrightarrow \quad 0<\frac{2 \beta^{2 k_{1}}\left(\beta^{2}-1\right)}{\left(\beta^{2 k+1}-1\right)(\beta+1)} .
\end{gathered}
$$

The last inequality holds since $\beta>1$ and therefore $x_{1}<x_{2 k}$.

Now we show that $x_{2}<-\alpha$. We note that from (3.8) follows that

$$
\frac{a\left(2 \beta^{2 k-2}-\beta^{2 k}+1\right)}{\left(\beta^{2 k+1}-1\right)(\beta+1)}<-\alpha .
$$

Then (considering (3.12))

$$
\begin{aligned}
x_{2} & =\beta x_{1}+a=\frac{a \beta^{2}\left(\beta^{2 k+1}-2 \beta^{2 k-1}-1\right)}{\left(\beta^{2 k+1}-1\right)(\beta+1)}-a \beta+a \\
& =\frac{a\left(\beta^{2 k+3}-2 \beta^{2 k+1}-\beta^{2}-\beta^{2 k+3}+\beta^{2 k+1}+\beta^{2}-1\right)}{\left(\beta^{2 k+1}-1\right)(\beta+1)}=\frac{a\left(-\beta^{2 k+1}-1\right)}{\left(\beta^{2 k+1}-1\right)(\beta+1)} \\
& =\frac{a\left(2 \beta^{2 k-2}-\beta^{2 k}+1-2 \beta^{2 k-2}+\beta^{2 k}-\beta^{2 k+1}-2\right)}{\left(\beta^{2 k+1}-1\right)(\beta+1)} \\
& <-\alpha-\frac{a\left(\beta^{2 k+1}-\beta^{2 k}+2 \beta^{2 k-2}+2\right)}{\left(\beta^{2 k+1}-1\right)(\beta+1)}<-\alpha .
\end{aligned}
$$

Similar as (3.11) it is possible to show that $x_{2 m}<x_{2 m-2}$ for all $2 \leq m \leq k$.

If the assumptions of Theorem 8 hold, the orbit $O\left(x_{0}\right)$ where $x_{0}$ is defined by (3.9) (or (3.10)), satisfies (3.3) and is a periodic orbit of period $2 k+1$ for (3.1). Since $\beta>1$ then by Theorem 2 the orbit $O\left(x_{0}\right)$ is an unstable periodic orbit of period $2 k+1$.

In both Theorem 7 and 8 we have found periodic orbits for (3.1) such that points of orbits are greater or equal to $\alpha$ or less or equal to $-\alpha$, i.e., the points of the orbit belong to external lines of the function $h$. For example, if $\beta=2$, $a=21, \alpha=3$ and $k=3$, then by Theorem 7 we can find $x_{0}=12 \frac{1}{3}$ which is a point of a periodic orbit of period 6 : $\left\{12 \frac{1}{3}, 3 \frac{2}{3},-13 \frac{2}{3},-6 \frac{1}{3}, 8 \frac{1}{3},-4 \frac{1}{3}\right\}$. But for these parameters there exists another orbit of period 6 : $\{4,-13,-5,11,1,2\}$ (see Figure 8).

An interesting fact is that if we use formula (3.5) to find the initial point of a periodic orbit in the case when inequality (3.3) is not satisfied it is possible that we detect a periodic orbit as well. For example, if $\beta=2$, $a=21, \alpha=9$ and $k=3$, then $x_{0}=12 \frac{1}{3}$ gives a periodic orbit of period 6: $\left\{12 \frac{1}{3}, 3 \frac{2}{3}, 7 \frac{1}{3}, 14 \frac{2}{3}, 8 \frac{1}{3}, 16 \frac{2}{3}\right\}$. But there exists another periodic orbit of period 6: $\{16,11,1,2,4,8\}$ (see Figure 9). 


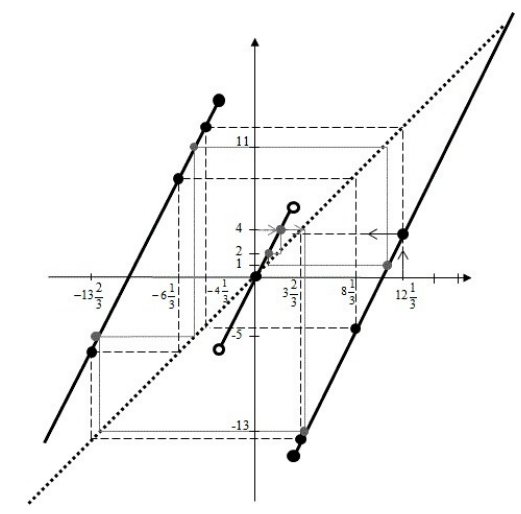

Figure 8. If $\beta=2, a=21, \alpha=3$, then by Theorem 7 there exists periodic orbit of period 6: $\left\{12 \frac{1}{3}, 3 \frac{2}{3},-13 \frac{2}{3},-6 \frac{1}{3}, 8 \frac{1}{3},-4 \frac{1}{3}\right\}$, but there exists another orbit of period 6 : $\{4,-13,-5,11,1,2\}$ too.

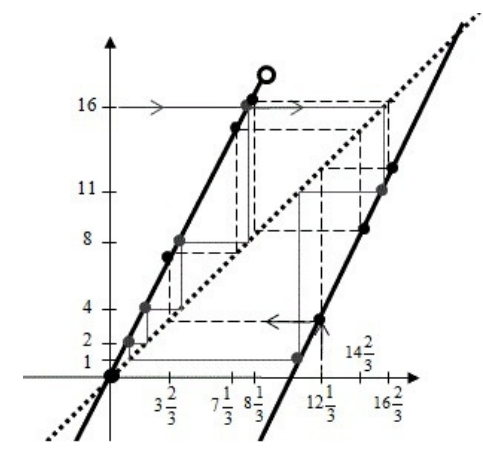

Figure 9. If $\beta=2, a=21, \alpha=9$, then there exists periodic orbits of period 6: $\left\{12 \frac{1}{3}, 3 \frac{2}{3}, 7 \frac{1}{3}, 14 \frac{2}{3}, 8 \frac{1}{3}, 16 \frac{2}{3}\right\}$ and $\{16,11,1,2,4,8\}$ — both with only positive points.

This means that by formulas (3.6) and (3.10) we can find some periodic orbits but not all. Furthermore we remark that in the situation with $\beta>1$ there exists infinitely many eventually periodic orbits.

\section{Chaotic Orbits}

More interesting cases involve bounded orbits that are not asymptotically or eventually periodic. If such orbit has a positive Lyapunov exponent (see [10, $12,14,15,16])$, then by definition [1] it is a chaotic orbit.

Definition 10. Let $f$ be a smooth map on $\mathbf{R}$. The Lyapunov exponent $\lambda\left(x_{0}\right)$ of the orbit $\left\{x_{0}, x_{1}, x_{2}, \ldots\right\}$ is defined as

$$
\lambda\left(x_{0}\right)=\lim _{n \rightarrow \infty} \frac{1}{n} \sum_{k=0}^{n-1} \ln \left|f^{\prime}\left(x_{k}\right)\right|,
$$

whenever the limit exists. 
Lyapunov exponent measure the infinitesimal exponential rate at which nearby orbits are moving apart. Note that if the application of the map to two nearby points leads to two points further apart, then the absolute value of the derivative of the map is greater than 1 when evaluated at these orbit points, and hence its logarithm is positive. If orbit points continue to diverge, then the rate of change of the logarithm of absolute values of derivatives is positive, and hence we have the presence of sensitive dependence on initial conditions. The Lyapunov exponent $\lambda\left(x_{0}\right)$ is a powerful experimental device to separate unstable, chaotic behavior from the one which is stable and predictable, and to measure these properties $[10,14]$.

Usually it is very difficult to calculate Lyapunov exponents exactly. One exception is a general tent map

$$
T_{p}(x)= \begin{cases}p x, & x \leq \frac{1}{2}, \\ p(1-x), & \frac{1}{2}<x .\end{cases}
$$

For any orbit of the tent map that does not contain a point $\frac{1}{2}$, we have $L\left(x_{0}\right)=$ $\ln p[12,15]$. For the logistic map $L(x)=4 x(1-x)$ Lyapunov exponent is the same as for $T_{2}(x)$, it is $\ln 2[12,15]$.

Function

$$
h(x)= \begin{cases}\beta x+a, & x \leq-\alpha, \\ \beta x, & -\alpha<x<\alpha, \\ \beta x-a, & \alpha \leq x,\end{cases}
$$

considered in this article is similar to the tent map. In this case $\lambda\left(x_{0}\right)=\ln \beta$ for all $x_{0}$ for which the orbit does not contain points $-\alpha$ and $\alpha$.

By [16] when the Lyapunov exponent is positive, this indicates that the system has sensitive dependence, when the Lyapunov exponent is negative, this indicates that the orbit is going to an attracting periodic orbit. In the case if $\beta<1$ then all orbits go to an attracting periodic point, also to a stationary point. If $\beta>1$ then system has sensitive dependence. We have observed in previous section that all periodic orbits are unstable. But usually with term "chaotic mapping" we understand much more. By the definition of R. Devaney [8] a function $f: X \rightarrow X((X, \rho)$ is a metric space) is chaotic if the function $f$ is topologically transitive, exhibits sensitive dependence on initial conditions and the set of periodic points of $f$ are dense in $X$. In this definition it is not essentially for the function to be continuous. Further we work with term "chaotic orbit" which is slightly another concept for chaotic behavior.

Definition 11. [1] Let $f$ be a map of the real line $\mathbf{R}$ and let $\left\{x_{0}, x_{1}, x_{2}, \ldots\right\}$ be a bounded orbit of $f$. The orbit is chaotic if

1. $\left\{x_{0}, x_{1}, x_{2}, \ldots\right\}$ is not asymptotically or eventually periodic,

2. the Lyapunov exponent $\lambda\left(x_{0}\right)$ is greater than zero.

By [1] for the tent map $T_{2}$ and doubling map $D(x)=2 x(\bmod 1)$ in the interval $[0,1]$ each orbit that forever avoids $\frac{1}{2}$ and is not asymptotically or eventually periodic is a chaotic orbit (with Lyapunov exponent $\ln 2$ ). Very similar to the doubling map is Baker's map $[9,10,15]$ or Saw-Tooth map [14] 
that differ from doubling map with a definition in points $\frac{1}{2}$ and 1 - values of function are equal to 0 and 1 . One special case of our model with parameters $\beta=2, \alpha=\frac{1}{2}$ and $a=1$ in the interval $[0,1]$ is equal to the considered Baker's mapping

$$
B(x)= \begin{cases}2 x, & 0 \leq x<\frac{1}{2}, \\ 2 x-1, & \frac{1}{2} \leq x \leq 1,\end{cases}
$$

therefore contains chaotic orbits.

In our model Lyapunov exponent $\lambda\left(x_{0}\right)$ is greater than zero. By Definition 11 we need to find bounded orbits which are not asymptotically or eventually periodic. In other words, we need to find an invariant set $A$ for the function $h$, then for every point of the set the orbit will be bounded. Here we consider some cases when exists invariant set and when does not exist.

If we want to find the invariant set $[A, B](A<B)$ that contains interval $[-\alpha, \alpha]$ then conditions should hold:

$$
\begin{gathered}
A \leq-\alpha \beta, \quad A \leq \alpha \beta-a, \quad A \leq \beta A+a, \\
-\alpha \beta+a \leq B, \quad \alpha \beta \leq B, \quad \beta B-a \leq B .
\end{gathered}
$$

From the third and sixth conditions follows that

$$
\frac{-a}{\beta-1} \leq A<B \leq \frac{a}{\beta-1},
$$

that is, the invariant set is located between the stationary states. Since here is too many parameters, it is difficult to establish all cases. One case is if $\frac{a}{\beta-1}=\alpha \beta$, then the invariant interval is $[-\alpha \beta, \alpha \beta]$.

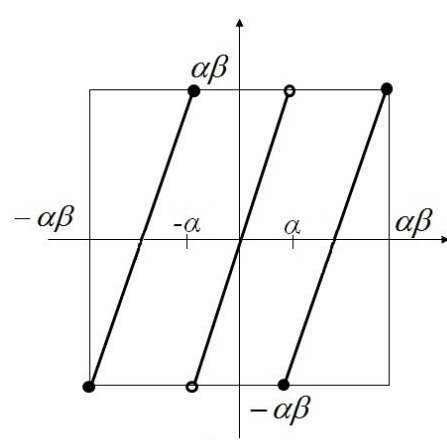

a)

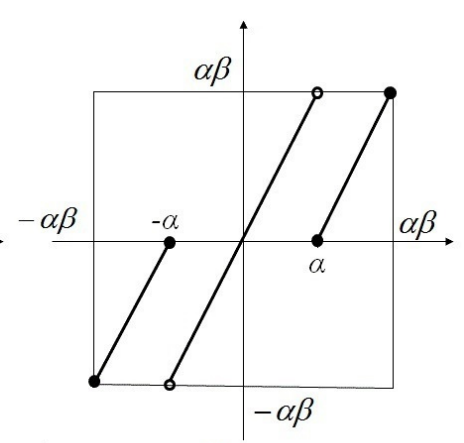

b)

Figure 10. $[-\alpha \beta, \alpha \beta]$ is invariant set of function $h$. a) $a=2 \alpha \beta$ b) $a=\alpha \beta$.

In Figure 10 we consider two situations with the invariant set $[-\alpha \beta, \alpha \beta]$. In the first case $a=2 \alpha \beta$, from this follows that $\frac{2 \alpha \beta}{\beta-1}=\alpha \beta$ therefore this situation is only when $\beta=3$. Similarly we obtain that in the second case $\beta=2, \alpha$ and $a$ are freely chosen. In the second case an invariant set is also $[0, \alpha \beta]$. This case is similar to Baker's map.

From the second case it follows that we can find an invariant set in the interval $\left[0, \frac{a}{\beta-1}\right]$. One situation is when

$$
0<\alpha \beta-a<\alpha \beta^{2}-a<\alpha \beta
$$




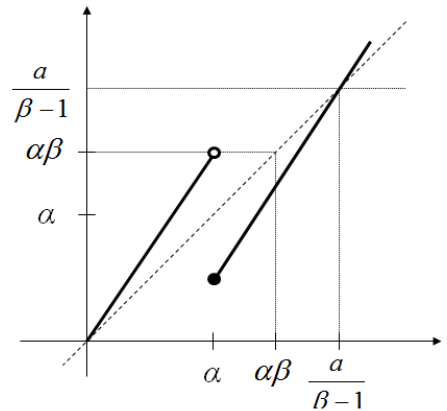

a)

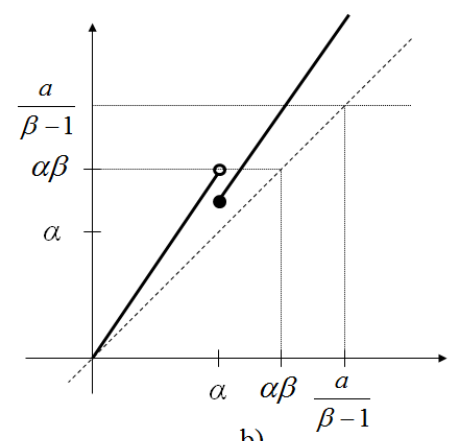

b)

Figure 11. a)Invariant set of function $h$ is interval $[0, B]$ where $B \in\left[\alpha \beta, \frac{a}{\beta-1}\right]$. b)For function $h$ does not exist invariant set.

then mapping $h$ is invariant in the set $[0, B]$ where $B \in\left[\alpha \beta, \frac{a}{\beta-1}\right]$ (see Figure $11 \mathrm{a})$ ). We conclude that in this situation for mapping $h$ exist chaotic orbits. Obviously that for a function in the form $y=c x+d$ does not exist an invariant set. Therefore the mapping $h$ does not have an invariant set if

$$
0<a<\alpha \beta-\alpha
$$

(see Figure $11 \mathrm{~b})$ ). This means that in this situation there is no chaotic orbits.

Therefore we can formulate a following theorem.

Theorem 9. There exists parameters $\beta, \alpha$ and a such that the function $h$ has chaotic orbits in $\left[\frac{-a}{\beta-1}, \frac{a}{\beta-1}\right]$.

\section{Conclusions}

In the paper we proved some results about the periodicity of solutions of difference equation (3.1) accordingly to parameters $\beta, \alpha$ and $a$ of the signal function. The main conclusions are

1) if $\beta=1$ then every point $x_{0} \in \mathbf{R}$ is a stationary state or an eventually stationary state or the orbit $O\left(x_{0}\right)$ is a periodic orbit of period 2 or eventually periodic orbit of period 2 ;

2 ) if $\beta<1$ then the point 0 is an unique stationary state, there exists periodic orbit of period $2\left\{\frac{-a}{\beta+1}, \frac{a}{\beta+1}\right\}$ and other orbits are asymptotically or eventually stationary state to 0 or asymptotically or eventually periodic orbit of period 2 , 3 ) if $\beta>1$ then the point 0 always is a stationary state but it is possible to have two more stationary states; there exists maximum three periodic orbits of period 2 but it is possible that there are no periodic orbits of period 2 ; for some parameters we have constructed periodic orbits of an arbitrary period and finally we have concluded that there exists parameters such that there exists chaotic orbits.

With respect to parameters our model has different behavior. By [19] $x$ denotes the activation level of a neuron. If one neuron works as the considered 
model then we can interpret a stationary state as an equilibrium state in which activation level is constant, then the periodic orbit means periodic changes of activation level. A chaotic orbit means not predictable changes of activation level but for the orbit that goes to infinity we cannot give a good interpretation (activation level grow in time without restriction).

We conclude that model (3.1) with the signal function (3.2) describes more general situation as considered in [22] (also [5, 18, 20,23, 24]).

Chaotic mappings can be applied, for example, in cryptography. Chaosbased image encryption has become one of efficient encryption methods. This is because chaotic maps have high sensitivity on the initial values and control parameters $[2,21]$. Similar functions as $h$ are used to study the power spectral density of signals with applications in telecommunications and transmission security [11].

\section{Acknowledgement}

This research was partially supported by the European Social Fund within the project Support for Doctoral Studies at University of Latvia and Latvian Council of Science research project 345/2012.

The authors are grateful to anonymous reviewers for their valuable comments and suggestions, which helped to enrich the content and improve the presentation of this paper.

\section{References}

[1] K.T. Alligood, T.D. Sauer and J.A. Yorke. CHAOS. An Introduction to Dynamical Systems. Springer-Verlag New York, Inc., 1997.

[2] G. Alvarez and S. Li. Breaking an encryption scheme based on chaotic baker map. Phys. Lett. A, 352:78-82, 2006.

http://dx.doi.org/10.1016/j.physleta.2005.11.055.

[3] A. Anisimova, M. Avotina and I. Bula. Periodic orbits of single neuron models with internal decay rate $0<\beta \leq 1$. Math. Model. Anal., 18:325-345, 2013. http://dx.doi.org/10.3846/13926292.2013.804462.

[4] F.A. Azevedo, L.R. Carvalho, L.T. Grinberg, J.M. Farfel, R.E. Ferretti, R.E. Leite, W.J. Filho, R. Lent and S. Herculano-Houzel. Equal numbers of neuronal and nonneuronal cells make the human brain an isometrically scaled-up primate brain. J. Comparative Neurology, 513:532-541, 2009. http://dx.doi.org/10.1002/cne.21974.

[5] Y. Chen. All solutions of a class of difference equations are truncated periodic. Appl. Math. Lett., 15:975-979, 2002. http://dx.doi.org/10.1016/S0893-9659(02)00072-1.

[6] R. Cheng. Oscillatory periodic solutions for two differential-difference equations arising in applications. Abstr. Appl. Anal., 2011:Article ID 635926, 12 pages, 2011. http://dx.doi.org/10.1155/2011/635926.

[7] S.N. Chow and H.O. Walther. Characteristic multipliers and stability of symmetric periodic solutions of $\dot{x}(t)=g(x(t-1))$. Trans. Amer. Math. Soc., 307:127142, 1988. http://dx.doi.org/10.1090/S0002-9947-1988-0936808-2. 
[8] R. Devaney. An Introduction to Chaotic Dynamical Systems. second ed., Addison-Wesley, 1989.

[9] S.N. Elaydi. An Introduction to Difference Equations. second ed., SpringerVerlag New York, Inc., 1999.

[10] S.N. Elaydi. Discrete Chaos. With Applications in Science and Engineering. second ed., Chapman \& Hall, CRC, 2008.

[11] K. Feltekh, Z.B. Jemaa, D. Fournier-Prunaret and S. Belghith. Border collision bifurcations and power spectral density of chaotic signals generated by one-dimensional discontinuous piecewise linear maps. Commun. Nonlinear Sci. Numer. Simul., 19:2771-2784, 2014.

http://dx.doi.org/10.1016/j.cnsns.2013.12.029.

[12] M.R.S. Kulenovic and O. Merino. Discrete Dynamical Systems and Difference Equations with Mathematica. Chapman \& Hall, CRC, 2002.

[13] R.D. Nussbaum. Uniqueness and nonuniqueness for periodic solutions of $x^{\prime}(t)=$ $-g(x(t-1))$. J. Differential Equations, 34:25-54, 1979. http://dx.doi.org/10.1016/0022-0396(79)90016-0.

[14] H.O. Peitgen, H. Juergen and D. Saupe. Chaos and Fractals. New Frontiers of Science. second ed., Springer-Verlag New York, Inc., 2004.

[15] C. Robinson. Dynamical Systems. Stability, Symbolic Dynamics, and Chaos. CRC Press, Inc., 1995.

[16] R.C. Robinson. An Introduction to Dynamical Systems. Continuous and Discrete. Pearson Education, 2004.

[17] H.O. Walther. Homoclinic solutions and chaos in $\dot{x}(t)=f(x(t-1))$. Nonlinear Anal., 5:775-788, 1981. http://dx.doi.org/10.1016/0362-546X(81)90052-3.

[18] Z. Wei, L. Huang and Y. Meng. Unboundedness and periodicity of solutions for a discrete-time network model of three neurons. Appl. Math. Model., 32:14631474, 2008. http://dx.doi.org/10.1016/j.apm.2007.06.016.

[19] J. Wu. Introduction to Neural Dynamics and Signal Transmission Delay. De Gruyter, Berlin, 2001.

[20] Z. Yuan, L. Huang and Y. Chen. Convergence and periodicity of solutions for a discrete-time network model of two neurons. Math. Comput. Model., 45:941-950, 2002. http://dx.doi.org/10.1016/S0895-7177(02)00061-4.

[21] Y. Zhou, L. Bao and C.L.P. Chen. A new 1d chaotic system for image encryption. Signal Processing, 97:172-182, 2014.

http://dx.doi.org/10.1016/j.sigpro.2013.10.034.

[22] Z. Zhou. Periodic orbits on discrete dynamical systems. Comput. Math. Appl., 45:1155-1161, 2003. http://dx.doi.org/10.1016/S0898-1221(03)00075-0.

[23] Z. Zhou and J. Wu. Stable periodic orbits in nonlinear discrete-time neural networks with delayed feedback. Comput. Math. Appl., 45:935-942, 2003. http://dx.doi.org/10.1016/S0898-1221(03)00066-X.

[24] H. Zhu and L. Huang. Dynamics of a class of nonlinear discrete-time neural networks. Comput. Math. Appl., 48:85-94, 2004.

http://dx.doi.org/10.1016/j.camwa.2004.01.006. 